\title{
Staatsrecht junger Nationalstaaten
}

Grundriß.- Hrsg. von der Akademie für Staats- und Rechtswissenschaft der DDR, der Karl Marx-Universität Leipzig und der Akademie der Wissenschaften der DDR, Staatsverlag der Deutschen Demokratischen Republik, Berlin, 1988, 300 S.

Die Staatsrechtswissenschaft der DDR ist organisiert in klar voneinander gesonderten Disziplinen, was sich auch in jeweils zu einer solchen Disziplin vorgelegten, regelmäßig kollektiv verfaßten Lehrbüchern ausdrückt. Unterschieden wird die "Wissenschaft vom Staatsrecht der sozialistischen Staaten" einerseits, der "bürgerlichen Staaten" andererseits. Hinzu tritt die "Wissenschaft vom Staatsrecht der jungen Nationalstaaten". Hierunter werden ausschließlich Staaten des afrikanischen und des asiatischen Raumes verstanden. Trotz des Akjektivs "jung", das gewöhnlich auf ein Datum der Unabhängigkeitserlangung nach dem 2. Weltkrieg abstellt, werden - angesichts vergleichbarer politischer und wirtschaftlicher Bedingungen - auch in diesem Sinne "alte" Staaten, wie z.B. Thailand, Afghanistan oder Iran einbezogen. Staaten des lateinamerikanischen Kontinents, die gleichfalls Objekt kolonialer Penetration waren und sich teilweise in weitgehender Problem- und Interessenidentität mit afrikanischen und asiatischen Staaten befinden, bleiben aber ausgeklammert. Von den Staatsformen her gedacht, liegt eine gesonderte Erfassung "junger" bzw. "ihnen ähnlicher" Staaten quer zu den eingangs schon genannten Kategorien "bürgerlich" und "sozialistisch". Deshalb findet sich auch innerhalb der Gruppe "junger Nationalstaaten" wiederum eine Aufteilung in solche "kapitalistischer Entwicklung" bzw. solche mit "revolutionärer Demokratie"; die trotz dieser grundlegenden Unterscheidung, die das Selbstverständnis der Wissenschaft in der DDR gebietet, vorhandenen Gemeinsamkeiten lassen es aber zu, an dem Oberbegriff "junger Nationalstaaten" festzuhalten.

So erklärt sich die Gliederung des vorliegenden, als "Grundriß" bezeichneten Lehrbuchs: Einem ersten Teil "Grundfragen des Staatsrechts junger Nationalstaaten" (S. 13-57), folgen die Kapitel "Der Staat in den Ländern kapitalistischer Entwicklung" (S. 61-175) sowie "Der revolutionär-demokratische Staat" (S. 179-286), beide fast exakt gleichen Umfangs. Beide Kapitel zu den Staaten unterschiedlichen Typs zerfallen je in eingehende allgemeine Teile (einmal "Grundzüge", einmal "Grundlagen" genannt) und sehr knappe Kurzdarstellungen des Staatsrechts exemplarisch ausgewählter Einzelstaaten: Als "kapitalistisch" firnnieren insoweit Indien, Malaysia, die Elfenbeinküste, Ägypten und Kuwait, als "revolutionärdemokratisch" Algerien, Madagaskar, Tansania, Kongo, Angola, Afghanistan und (Süd-) Jemen.

Der vor die Klammer gezogene erste Teil des Buchs bringt einen historischen Abriß, in dem - wie gehabt - von dem "Fanal, das die Sowjetmacht auch mit der nationalen Befreiung unterdrückter Völker und mit der Herstellung eines neuen Typs internationaler Beziehungen schuf" und ähnlichem die Rede ist. Die Darstellung verwendet bekannte Theoreme und Kategorien, berücksichtigt im wesentlichen eigene Arbeiten von Wissenschaftlem der 
DDR, solchen aus der Sojwetunion ${ }^{1}$, aber gelegentlich auch aus dem westlichen Ausland, eingebunden in Marx-, Lenin-, Gorbatschow- und Honecker-Zitate sowie Parteitagsdokumente. Ziel der Darstellung ist der Nachweis, daß trotz der differenten Einzelbedingungen in den jeweiligen, einstmals kolonialer Herrschaft unterworfenen Territorien und trotz der Unterschiedlichkeit der nach Abschluß der die Kolonialisierung eingeschlossenen Wege die gegenwärtige Situation von Staat und Recht durchweg eine solche sei, die die Dogmen der marxistisch-leninistischen Staats- und Rechtstheorie in ihrer Gestalt vor Anlaufen der Neuorientierungsversuche in der Sowjetunion bestätigt2. Allerdings würden die Formen staatlicher Organisation und die "Grundrichtungen der Tätigkeit der staatlichen Organe" "zunehmend" - durch die Spezifika des jeweils gewählten Entwicklungsweges geprägt.

Im Abschnitt über "afro-asiatische Staaten kapitalistischer Entwicklung" - es seien dies derzeit etwa 80 Staaten - wird zunächst der definitorische Ausgangspunkt präzisiert: "Kapitalistische" Entwicklungsländer seien solche, die "neo-kolonialer Ausbeutung durch transnationale Konzerne und imperialistische Staaten" unterlägen. Herrschende, jedenfalls die Herrschaft prägende Klasse sei jeweils die Bourgeoisie; das theoretische Etikett lautet: "Staat eines unterschiedlich fortgeschrittenen Ubergangs zum bürgerlichen Typ". Die besondere Relevanz des staatlichen Wirtschaftssektors wird als Charakteristikum "kapitalistischer" Entwicklungsländer in Asien und Afrika herausgearbeitet, sodann werden weitere Gruppierungen versucht, so nach dem sozial-ökonomischen Entwicklungsstand und den Unterschieden im "Klassenwesen", ehe die Rolle politischer Parteien, einzelne Regierungsformen und Elemente des Staatsaufbaus analysiert und typologisiert werden. Eher deskriptiv ausgerichtet ist ein Überblick über vorhandene Verfassungen (auch: zur Verfassungskontinuität), über Grundrechtsverbürgungen und einzlne Staatsorgane einschließlich der Justiz. Dabei wird oft anschaulich mit Beispielen gearbeitet. In Ansehung der Grundrechte tut sich die Darstellung in gewohnter Weise schwer, weil an der Prämisse festgehalten wird, in den Verfassungsordnungen "bürgerlicher" Staaten gewährleistete Grundrechte unterlägen "gewöhnlich erheblicher Einschränkung" (gemeint: in stärkerem Maße als in Staaten sozialistischen Typs); diese Prämisse wird auf "kapitalistische" Entwicklungsländer übertragen und verschließt damit von vomherein den Blick auf die entwicklungsländerspezifische Begrenztheit des Grundrechtsschutzes, seine Unterschiede zu europäischamerikanisch ausgelegtem Grundrechtsschutz und seine Unterschiede innerhalb von Entwicklungsländern.

Die Einzelländerstudien wählen dann in überraschender Weise aus: Alle fünf untersuchten Staaten waren zuvor - nach der erwähnten Klassifizierung - der Gruppe von Staaten mit "mittlerem Entwicklungsniveau" (nämlich Kuwait und die Elfenbeinküste) bzw. mit "relativ hohem Niveau" zugeordnet worden (Indien, Ägypten, Malaysia), keine aber den Staaten mit "sehr geringem Entwicklungsstand" (wie z.B. Mauretanien) oder mit "hohem Niveau"

1 Vgl. W.N. Denissow, Rechtssysteme in Entwicklungsländem, 1981, dazu meine Rezension in: Verf assung und Recht in Übersee 16 (1983), S. 460-461.

2 Repräsentativ: Marxistisch-leninistische Staats- und Rechtstheorie, Lehrbuch, 1980. 
der Produktivkräfte (wie Südkorea). Auch über die innere Schlüssigkeit solcher Einordnung ließe sich natürlich streiten.

Die Länderberichte selbst beschränken sich im wesentlichen auf die Mitteilung politischer Grundfakten und die zusammenfassende Schilderung von Verfassungsinhalten. Westliche Literatur ist in geringem Umfang, oft mit älteren Werken berücksichtigt, aber kaum eigentlich eingearbeitet. Das gilt auch für Beiträge aus den behandelten Staaten selbst; das Vorwort vermerkt hierzu, dem Bemühen um die Berücksichtigung von Stimmen aus Entwicklungsländern habe - "unter anderem" - entgegengewirkt, daß sich in zahlreichen dieser Staaten eine "nationale Staats- und Rechtswissenschaft" noch nicht habe herausbilden können - was etwa für Indien eine absurde Feststellung wäre. Dennoch kann auch der Indienbeitrag keine Auseinandersetzung mit den Stimmen der indischen Verfassungsrechtswissenschaft bieten.

Der Abschnitt des Buches über "revolutionär-demokratische" Entwicklungsländer behandelt diejenigen Staaten, die die Sowjetunion und die DDR ihrem "Lager" zuordnen. Mit gebührender Sympathie werden deshalb die sich im Recht und Verfassung ausdrückenden Bemühungen dieser Staaten und die objektiven Schwierigkeiten, auf die sie treffen, geschildert. Den "jungen Volksrepubliken" wird in besonderem Maße bescheinigt, nach dem Abbau von Unterentwicklung zu streben und "langfristig Voraussetzungen für eine sozialistische Perspektive zu schaffen". Zur Grundrechtsfrage heißt es, "Grundrechtsaussagen" würden "nicht unabhängig, sondern in ... Beziehung zum sozialen Wesen und zu den sozialen Zielen der Staatsmacht erfolgen"; sie würden "in ihrer Einheit" mit den "Pflichten der Bürger" geregelt. Auch hier ist also keine Abkehr von der strikten Reduktion des Grundrechtsschutzes auf Programmatik zu erkennen, wie sie sich ja ansatzweise schon zeigt in neueren Stellungnahmen von sow jetischen Wissenschaftlern, auch wenn dies im Schrifttum noch kaum dokumentiert ist. Noch stärker als im Parallelabschnitt zu den "kapitaltistischen Entwicklungsländern" wird hier übrigens mit Details und Beispielen der Theoriefluß aufgelockert, was der Darstellung zugute kommt.

Die Einzelbeiträge zum Staatsrecht Algeriens, Madagaskars, Tansanias, Kongos, Angolas, Afghanistans und (Süd-) Jemens haben ähnlichen Zuschnitt wie die bereits erwähnten Länderberichte zur erstbehandelten Staatengruppe3. Hier fehlt es besonders stark an konkreter Realitätsbetrachtung, die Gesamtverfassung eines Staates soll erfaßt werden an Hand seiner geschriebenen Verfassung bzw. der von der Staatsmacht gesetzten normativen

3 Auffällig das Fehlen Mosambiks, das exemplarisch behandelt worden ist in H. GraflD. Joseph, Volksrepublik Mocambique - Werden und Wachsen eines jungen Staates, 1984, vgl. meine Rezension in: Verfassung und Recht in Übersee 18 (1985), S. 233-234. - H. Graf ist verantwortlich auch für einen großen Teil des vorliegenden Bandes. Die neueste Umorientierung in Mosambik läßt die DDR-Wissenschaftler wohl zweifeln, ob dieser Staat noch als "revolutionär-demokratisch" einzuordnen ist. 
Programmatik ${ }^{4}$. Ohne jegliche Einbeziehung verfassungsrechtswissenschaftlicher oder gar sozialwissenschaftlicher Diskussion bleibt etwa der Beitrag über Tansania. Die afghanische Malaise wird noch ohne jeden Beigeschmack zwischenzeitlich geäußerter sowjetischer Selbstkritik weggeblendet; sie klingt an in dem Hinweis, auf "mehrfach geäußerte Bitte" der afghanischen Führung habe die Sowjetunion sich bereitgefunden, "die revolutionäre Umgestaltung militärisch sichern zu helfen".

Das Lehrbuch ist orthodox, und es ist (nach dem Vorwort: bewußt) selektiv. Zu Iran findet sich beispielsweise in der gesamten Darstellung nur das Wort, dieses Land habe sich einmal in "halbkolonialer Abhängigkeit" gefunden. Libyen findet lediglich Erwähnung mit dem Hinweis, es sei von einem "charakteristischen" Schicksal für eine frühere Monarchie "ereilt" worden. Der Brückenschlag zur verfassungsrechtswissenschaftlichen Entwicklungsländerforschung außerhalb des sowjetischen Einflußbereichs wird nach wie vor nicht gesucht, anders also als beispielsweise im Bereich der Wissenschaft von der Internationalen Politik und in der Völkerrechtswissenschaft 5 . Dennoch ist das Erscheinen dieses Buches auch außerhalb jenes Einflußbereichs zu begrüßen. Es dokumentiert die Perspektiven und den Diskussionsstand in der DDR, und in der Sache selbst hält es für den Interessierten manche Einzelheit bereit. Ein sorgfältig gestaltetes Register vermittelt den Zugang.

Philip Kunig

\section{Ingrid Detter de Lupis \\ The Concept of International Law \\ Norstedts Förlag, Stockholm, 1987, 145 Seiten.}

Die von McDougal begründete "soziologische Schule" begriff das Völkerrecht als einen Prozeß, als ein "Interaktionsgefüge" zwischen Akteuren der verschiedensten Art und neigt folglich dazu, dem Völkerrecht letztlich jede rechtliche Verbindlichkeit abzusprechen. Im Zeitalter des Kooperationsvölkerrechts, in welchem jeder Mitgliedstaat der Völkerrechtsgemeinschaft sich auf das Verhalten der anderen Mitglieder verlassen können muß und in welchem die staatlichen Aufgaben nur noch durch das Zusammenwirken aller Staaten durchgesetzt werden können, erscheint ein solcher "approach" gefährlich, weil er das

4 Insofem ergiebiger: G. Brehme / H. Baumann u.a., Verfassung und Verfassungswirklichkeit in Ländern Asiens und Afrikas, 1984, vgl. meine Rezension in: Verfassung und Recht in Übersee, a.a.O. (Fn. 3).

5 Vgl. den Band "Abrüstung - Überlebensf ragen der Menschheit" hrsg. vom Institut für Intemationale Politik und Wirtschaft der DDR, 1987; beharrend aber "Völkerrecht, Grundriß", 2. Aufl. 1988 - beide Werke besprochen von H. Weber, in: Verfassung und Recht in Übersee 22 (1989), S. 230235. 